\title{
DISTRIBUTED BLIND DECONVOLUTION OF SEISMIC SIGNALS UNDER SPARSITY CONSTRAINTS IN SENSOR NETWORKS
}

\author{
Ban-Sok Shin and Dmitriy Shutin \\ Institute of Communications and Navigation, German Aerospace Center \\ E-Mail: \{ban-sok.shin, dmitriy.shutin\}@dlr.de
}

\begin{abstract}
This work proposes an iterative algorithm for distributed deconvolution of seismic signals for a reflectivity survey by a network of sensors. Distributed deconvolution is particularly relevant for a subsurface exploration by sensor networks or swarms of mobile robots. We envision such an exploration methodology by multiple mobile agents for future explorations of a planet's subsurface. The proposed scheme consists of two steps: distributed estimation of the seismic wavelet, followed by a local estimation of the reflectivity. Both steps are realized using alternating directions method of multipliers algorithms where we exploit sparsity in the reflectivity. The performance of the scheme is compared to state-of-the-art sparse multichannel blind deconvolution of seismic data and is found to be comparable or even superior.
\end{abstract}

Index Terms - Blind deconvolution, distributed processing, reflection seismology, sparse recovery

\section{INTRODUCTION}

Seismic surveys such as reflection seismology use multiple seismic sensors or geophones in order to record the seismic waves that are reflected at underground layers. Based on the received waves the subsurface of a given area can be reconstructed using signal processing algorithms [1]. However, most of these processing algorithms assume a centralized operation, i.e., all measurement data is available at a central entity. In contrast to that, we envision a distributed operation of the subsurface reconstruction that is done within a network of geophones. To this end, each geophone is additionally equipped with algorithmic and communication capabilities that allow a data processing and exchange with other geophones in the network. Such an architecture is particularly relevant for the exploration of a planet's subsurface such as on Mars or Moon [2] where an autonomous operation is required and a central processing entity might not be available. In this paper, we aim at taking a first step into a distributed seismic exploration method by focusing on the development of a distributed deconvolution method for seismic signals. Deconvolution of seismic signals is an important processing step in seismic data processing that removes unwanted distortions in the measurements.

A common survey methodology in seismic exploration is the reflectivity survey. Here, a seismic source such as a sledge hammer or air gun is activated and reflected waves at underground layers are recorded at multiple geophones that are placed over an area of interest. This measurement process can be modeled by a single-inputmultiple-output system where the source signal is convolved with the

The authors would like to thank Dr. Naveed Iqbal for providing a routine to generate the synthetic seismic data used in this paper. impulse responses of multiple reflectivity paths. Each such reflectivity path describes the reflectivity profile of the specific ray path the seismic wave travels from the source to the receiver. The goal is to recover the hidden impulse response of the reflectivity profile by deconvolving the measurements of the geophones with the seismic source signal. However, the seismic source or wavelet is usually unknown posing challenges to the deconvolution. Hence, blind deconvolution algorithms are needed that recover both the source signal and the reflectivity profile. In the past, a lot of research has been contributed to the field of blind seismic deconvolution, see [3, 4] and references therein. In particular, sparse multichannel blind deconvolution (SMBD) methods have shown promising performance results for the reflectivity estimation. The recent work of [4] uses an iterative algorithm that alternates between wavelet and reflectivity estimation. The wavelet estimation is conducted in the frequency domain whereas the reflectivity estimation is done in the time domain to exploit sparsity in the reflectivity's impulse response.

However, all of these state-of-the-art deconvolution methods consider a centralized operation. Our paper proposes a distributed blind deconvolution algorithm inspired by [4]. Both wavelet and reflectivity estimation are conducted in a distributed fashion within the seismic network such that each sensor in the network obtains an estimate of the impulse response of its corresponding reflectivity. To this end, we propose a distributed wavelet estimation within the seismic network using the alternating direction method of multipliers. Based on the estimated wavelet each sensor then solves an $\ell_{1}$-regularized problem locally to obtain a sparse estimate of the impulse response of its reflectivity. Performance evaluations indicate that the proposed scheme performs similar to centralized state-of-the-art blind deconvolution schemes.

\section{SYSTEM MODEL}

We consider a network of $J$ connected sensors each equipped with a geophone that measures a seismic trace at its corresponding position. We describe the network topology by a graph $\mathcal{G}=\{\mathcal{J}, \mathcal{E}\}$ with a set of nodes $\mathcal{J}=\{1,2, \ldots, J\}$ and a set of edges $\mathcal{E}=\{(j, i) \mid j, i \in$ $\mathcal{J}, j \neq i\}$. With $\mathcal{N}_{j}$ we denote a neighborhood of the sensor $j$ - a set of all sensors directly connected to sensor $j$, including sensor $j$ itself. In addition, we assume that the network graph is undirected and strongly connected [5].

It is common to model the acquisition of a seismic trace via a convolution of a seismic source wavelet $\boldsymbol{w}$ and the impulse response $\boldsymbol{h}_{j}$ that models the subsurface reflection between the source and the receiver $j$ [1]. The impulse response $\boldsymbol{h}_{j}$ is typically termed reflectivity. It characterizes the reflections occurring as the sound ray passes between source and the receiver by reflecting off different subsurface layers. Thus, it is the reflectivity that is important for quantifying the 
subsurface under consideration. Unfortunately, due to the non-ideal form of the seismic source, the reflectivity profile of the subsurface is blurred, which makes a reliable subsurface reconstruction difficult. Therefore, the goal of seismic deconvolution is to remove the influence of the seismic source on the seismic traces in order to infer "a clean" reflectivity. However, both source wavelet and reflectivity are usually unknown such that blind approaches are required. As proposed by [4] one can separate the blind deconvolution into a stage of wavelet estimation and reflectivity estimation. We make use of the same approach here but extend it for distributed operation. However, for wavelet estimation and reflectivity estimation we use different formulations of a system model which we present in the following.

\subsection{System Model for Wavelet Estimation}

Each sensor $j$ acquires a noisy seismic trace $\boldsymbol{d}_{j}$ which can be described by a convolution between a discrete time source wavelet $\boldsymbol{w}=\left[w(0), w(1), \ldots, w\left(L_{\mathrm{W}}-1\right)\right]^{\top} \in \mathbb{R}^{L_{\mathrm{W}}}$ and the reflectivity $\boldsymbol{h}_{j}=\left[h_{j}(0), h_{j}(1), \ldots, h_{j}\left(L_{\mathrm{R}}-1\right)\right]^{\top} \in \mathbb{R}^{L_{\mathrm{R}}}$ :

$$
d_{j}(l)=w(l) * h_{j}(l)+n_{j}(l)=\sum_{\nu=0}^{L_{\mathrm{W}}} h_{j}(l-\nu) w(\nu)+n_{j}(l),
$$

where $l=0, \ldots, L_{\mathrm{W}}+L_{\mathrm{R}}-1$. In matrix-vector notation, the seismic trace $\boldsymbol{d}_{j} \in \mathbb{R}^{L_{\mathrm{W}}+L_{\mathrm{R}}-1}$ at sensor $j$ can be formulated via

$$
\boldsymbol{d}_{j}=\boldsymbol{H}_{j} \boldsymbol{w}+\boldsymbol{n}_{j}, \quad j \in \mathcal{J},
$$

where $\boldsymbol{n}_{j} \in \mathbb{R}^{L_{\mathrm{W}}+L_{\mathrm{R}}-1}$ contains noise samples. Matrix $\boldsymbol{H}_{j} \in$ $\mathbb{R}^{\left(L_{\mathrm{W}}+L_{\mathrm{R}}-1\right) \times L_{\mathrm{W}}}$ is the convolution matrix of the reflectivity $\boldsymbol{h}_{j}$ at sensor $j$. Finally, we can stack the seismic traces of all $J$ sensors into a vector $\boldsymbol{d}=\left[\boldsymbol{d}_{1}^{\top}, \ldots, \boldsymbol{d}_{J}^{\top}\right]^{\top} \in \mathbb{R}^{J\left(L_{\mathrm{W}}+L_{\mathrm{R}}-1\right)}$ and all $J$ reflectivity matrices $\boldsymbol{H}_{j}$ into $\boldsymbol{H}=\left[\boldsymbol{H}_{1}^{\top}, \boldsymbol{H}_{2}^{\top}, \ldots, \boldsymbol{H}_{J}^{\top}\right]^{\top}$ which allows to reformulate model (2) as

$$
\boldsymbol{d}=\boldsymbol{H} \boldsymbol{w}+\boldsymbol{n}
$$

with $\boldsymbol{n}=\left[\boldsymbol{n}_{1}^{\top}, \ldots, \boldsymbol{n}_{J}^{\top}\right] \in \mathbb{R}^{J\left(L_{\mathrm{W}}+L_{\mathrm{R}}-1\right)}$. We assume white Gaussian noise with covariance matrix $\boldsymbol{\Sigma}=\operatorname{blkdiag}\left(\sigma_{1}^{2} \boldsymbol{I}, \ldots, \sigma_{J}^{2} \boldsymbol{I}\right)$ of dimension $J\left(L_{\mathrm{W}}+L_{\mathrm{R}}-1\right) \times J\left(L_{\mathrm{W}}+L_{\mathrm{R}}-1\right)$, where blkdiag builds a block-diagonal matrix and $\sigma_{j}^{2}$ is the noise variance at sensor $j$. It should be noted that each seismic trace $\boldsymbol{d}_{j}$ is generated by the same source wavelet $\boldsymbol{w}$. This fact will be later exploited to enable a distributed estimation of the wavelet $\boldsymbol{w}$.

\subsection{System Model for Reflectivity Estimation}

Inspecting (2) one observes that it can be also formulated in terms of a convolution matrix for the wavelet $\boldsymbol{w}$. In this case, the seismic trace $\boldsymbol{d}_{j}$ per sensor $j$ is the result of

$$
\boldsymbol{d}_{j}=\boldsymbol{W} \boldsymbol{h}_{j}+\boldsymbol{n}_{j}, \quad \forall j \in \mathcal{J} .
$$

Matrix $\boldsymbol{W} \in \mathbb{R}^{\left(L_{\mathrm{W}}+L_{\mathrm{R}}-1\right) \times L_{\mathrm{R}}}$ is the convolution matrix of the wavelet $\boldsymbol{w}$. In case $\boldsymbol{W}$ is available at sensor $j$ (4) directly enables the formulation of an optimization problem to recover the reflectivity $\boldsymbol{h}_{j}$ locally at each sensor $j$.

\section{DISTRIBUTED SPARSE BLIND DECONVOLUTION}

Based on the system models introduced in Section 2 we aim at estimating both the source wavelet $\boldsymbol{w}$ and the reflectivity vectors $\boldsymbol{h}_{j}$ in a distributed fashion within the network. As proposed in [4] for the centralized case, we can separate the problem into two steps: one step is an estimation of the wavelet $\boldsymbol{w}$ and the other one is the estimation of the reflectivity $\boldsymbol{h}_{j}$. Let us first introduce the wavelet estimation.

\subsection{Wavelet Estimation}

\subsubsection{Initialization of Reflectivities}

For the estimation of the wavelet $\boldsymbol{w}$ we rely on system model (3). However, to solve for $\boldsymbol{w}$ we need estimates of the reflectivities $\boldsymbol{h}_{1}, \ldots, \boldsymbol{h}_{J}$ contained in $\boldsymbol{H}$. To obtain initial estimates of the reflectivities Iqbal et. al. proposed the use of a peak locator per seismic trace $\boldsymbol{d}_{j}$ [4]. The motivation of this approach is to provide a rough estimate of the reflectivities that enables an initial wavelet estimation which in turn is used later to improve the reflectivity estimation. We use the same approach here to get an initial reflectivity estimate $\widehat{\boldsymbol{h}}_{j}$ at each sensor $j$. Based on these estimates we can construct a matrix $\widehat{\boldsymbol{H}}$ as an estimate of the stacked convolution matrix $\boldsymbol{H}$ in (3).

However, to obtain an appropriate initial estimate of the reflectivities we need to take into account the peak position of the wavelet $\boldsymbol{w}$. Since the source wavelet $\boldsymbol{w}$ is a modeled as a casual FIR system, its peak does not lie at the 0 -th sample but is shifted in positive time by $l_{\text {peak }}$ samples. Therefore, when convolving $\boldsymbol{w}$ with the reflectivity $\boldsymbol{h}_{j}$ the peaks in the seismic trace $\boldsymbol{d}_{j}$ are not aligned to the original peak positions but shifted by $l_{\text {peak }}$ samples as well. Hence, if we apply a peak locator on the seismic trace $\boldsymbol{d}_{j}$ the original peaks in $\boldsymbol{h}_{j}$ will be shifted by $l_{\text {peak }}$ in the initial reflectivity estimate $\widehat{\boldsymbol{h}}_{j}$. This offset will eventually lead to a corrupted wavelet estimate. Thus to enable an appropriate wavelet estimation, after applying the peak locator on the trace $\boldsymbol{d}_{j}$ the located peaks need to be shifted back in time by $l_{\text {peak }}$ samples. In reality this information needs to be estimated appropriately. For validation purposes, we assume that we know $l_{\text {peak }}$ perfectly at this stage. The initial estimate of each reflectivity is then modified as follows:

$$
\widehat{\boldsymbol{h}}_{j} \leftarrow\left[\widehat{h}_{j}\left(l_{\text {peak }}\right), \widehat{h}_{j}\left(1+l_{\text {peak }}\right), \ldots, \widehat{h}_{j}\left(L_{\mathrm{R}}+l_{\text {peak }}\right)\right],
$$

where the last $l_{\text {peak }}$ entries in $\widehat{\boldsymbol{h}}_{j}$ are set to 0 .

\subsubsection{Centralized Operation}

Using the estimate $\widehat{\boldsymbol{H}}$ of the convolution matrix of all reflectivities, we can formulate a regularized least squares (LS) problem for the wavelet $\boldsymbol{w}$ :

$$
\boldsymbol{w}_{\mathrm{LS}}=\underset{\boldsymbol{w}}{\operatorname{argmin}}\|\boldsymbol{d}-\widehat{\boldsymbol{H}} \boldsymbol{w}\|_{\boldsymbol{\Sigma}^{-1}}^{2}+\lambda_{\ell 2}\|\boldsymbol{w}\|_{2}^{2}
$$

We employ $\boldsymbol{\Sigma}^{-1}$ in the vector norm in (6) to achieve a noisedependent regularization. The variable $\lambda_{\ell 2}$ is a Tikhonov regularization parameter that is used to enhance numerical stability of the solution and to mitigate the possibility of an ill-posed problem. Solution to (6) is readily given in closed form as

$$
\boldsymbol{w}_{\mathrm{LS}}=\left(\sum_{j=1}^{J} \widehat{\boldsymbol{H}}_{j}^{\mathrm{\top}} \widehat{\boldsymbol{H}}_{j}+\sigma_{j}^{2} \lambda_{\ell 2} \boldsymbol{I}\right)^{-1} \sum_{j=1}^{J} \widehat{\boldsymbol{H}}_{j}^{\top} \boldsymbol{d}_{j} .
$$

Note that solution $\boldsymbol{w}_{\text {LS }}$ requires access to all estimated reflectivities $\left\{\widehat{\boldsymbol{h}}_{j}\right\}_{j=1}^{J}$ and seismic traces $\left\{\boldsymbol{d}_{j}\right\}_{j=1}^{J}$. As such (7) describes a centralized solution. For our considerations it will serve as the benchmark scheme for the distributed wavelet estimation. 


\subsubsection{Distributed Operation}

To estimate $\boldsymbol{w}$ in a distributed fashion, we begin with (6) but introduce individual wavelet estimates $\boldsymbol{w}_{j} \in \mathbb{R}^{L_{\mathrm{W}}}$ per sensor $j$. This allows us to reformulate (6) as the following LS estimation problem that is more suitable for distributed optimization:

$$
\begin{aligned}
& \min _{\left\{\boldsymbol{w}_{j}\right\}_{j=1}^{J}}\left\{\sum_{j=1}^{J} \frac{1}{2 \sigma_{j}^{2}}\left\|\boldsymbol{d}_{j}-\widehat{\boldsymbol{H}}_{j} \boldsymbol{w}_{j}\right\|_{2}^{2}+\frac{\lambda_{\ell 2}}{2 J}\left\|\boldsymbol{w}_{j}\right\|_{2}^{2}\right\} \\
& \text { s.t. } \boldsymbol{w}_{j}=\boldsymbol{w}_{i}, \quad \forall i \in \mathcal{N}_{j} .
\end{aligned}
$$

The factor $1 / \sigma_{j}^{2}$ originates from the noise-dependent regularization via $\boldsymbol{\Sigma}^{-1}$. Compared to (6) we separated the squared norms into a sum over the sensor-specific squared norms. In addition, we included a consensus constraint ( $8 b)$ to enforce that each sensor converges to the same wavelet estimate. The constraint ensures that the estimate of the sensor $j$ and sensors in its neighborhood $\mathcal{N}_{j}$ coincide. Since the network is assumed to be strongly connected, this ensures a network-wide consensus on the wavelet estimate.

To solve the optimization problem (8) we rely on the alternating direction method of multipliers (ADMM) framework [6]. To this end, we introduce an additional variable $\boldsymbol{z} \in \mathbb{R}^{L_{\mathrm{W}}}$ that is used in the regularization term and reformulate problem (8) such that it matches the ADMM framework:

$$
\begin{aligned}
& \min _{\left\{\boldsymbol{w}_{j}, \boldsymbol{z}\right\}_{j=1}^{J}}\left\{\sum_{j=1}^{J} \frac{1}{2 \sigma_{j}^{2}}\left\|\boldsymbol{d}_{j}-\widehat{\boldsymbol{H}}_{j} \boldsymbol{w}_{j}\right\|_{2}^{2}+\frac{\lambda_{\ell 2}}{2}\|\boldsymbol{z}\|_{2}^{2}\right\} \\
& \text { s.t. } \boldsymbol{w}_{j}=\boldsymbol{z}, \quad \forall j \in \mathcal{J} .
\end{aligned}
$$

Constraint (9b) guarantees that the consensus constraint (8b) is adopted via the additional variable $\boldsymbol{z}$. Solving (9) via ADMM leads to the following set of update equations:

$$
\begin{aligned}
\boldsymbol{w}_{j}^{[k+1]} & =\left(\widehat{\boldsymbol{H}}_{j}^{\top} \widehat{\boldsymbol{H}}_{j}+\sigma_{j}^{2} \rho_{\mathrm{W}} \boldsymbol{I}\right)^{-1}\left(\widehat{\boldsymbol{H}}_{j}^{\top} \boldsymbol{d}_{j}+\sigma_{j}^{2} \rho_{\mathrm{W}}\left(\boldsymbol{z}^{[k]}-\boldsymbol{u}_{j}^{[k]}\right)\right) \\
\boldsymbol{z}^{[k+1]} & =\frac{J \rho_{\mathrm{W}}}{\lambda_{\ell 2}+J \rho_{\mathrm{W}}}\left(\overline{\boldsymbol{w}}^{[k+1]}+\overline{\boldsymbol{u}}^{[k]}\right) \\
\boldsymbol{u}_{j}^{[k+1]} & =\boldsymbol{u}_{j}^{[k]}+\boldsymbol{w}_{j}^{[k+1]}-\boldsymbol{z}^{[k+1]}
\end{aligned}
$$

where the subscript $[k]$ denotes the ADMM iteration index, $\rho_{\mathrm{W}}>0$ is the regularization parameter for ADMM augmented Lagrangian, and $\overline{\boldsymbol{w}}^{[k]}=\frac{1}{J} \sum_{j=1}^{J} \boldsymbol{w}_{j}^{[k]}$ and $\overline{\boldsymbol{u}}^{[k]}=\frac{1}{J} \sum_{j=1}^{J} \boldsymbol{u}_{j}^{[k]}$ are the averaged wavelet estimate and the averaged Lagrange multiplier in the network, respectively. The averaged quantities can be obtained by each node via average consensus sharing [7]. However, to obtain the exact same averaged quantities at each node via average consensus multiple iterations are again required. To avoid another iterative stage we use only one consensus averaging step at each sensor $j$ per iteration $k$ to obtain estimates of $\overline{\boldsymbol{w}}^{[k]}$ and $\overline{\boldsymbol{u}}^{[k]}$. Thus, each sensor $j$ computes its own averaged $\overline{\boldsymbol{w}}_{j}^{[k]}$ and $\overline{\boldsymbol{u}}_{j}^{[k]}$ via

$$
\overline{\boldsymbol{w}}_{j}^{[k]}=\frac{1}{J} \sum_{i \in \mathcal{N}_{j}} \boldsymbol{w}_{i}^{[k]}, \quad \overline{\boldsymbol{u}}_{j}^{[k]}=\frac{1}{J} \sum_{i \in \mathcal{N}_{j}} \boldsymbol{u}_{i}^{[k]} .
$$

In our experiments we have found that one consensus step is sufficient to obtain an appropriate wavelet estimate. According to (11) only the neighboring wavelet estimates $\boldsymbol{w}_{i}^{[k]}$ need to be exchanged among the sensors per iteration $k$.

To further improve the wavelet estimate, we apply a low pass filter on the estimated wavelet $\boldsymbol{w}_{j}^{[k]}$ at each sensor $j$. Usually, the seismic source wavelet has a band-limited spectrum such that we can filter out high-frequency noise. For our considerations here, we apply a low pass filter with cut-off frequency $f_{\mathrm{LP}}=130 \mathrm{~Hz}$.

\subsection{Local Estimation of Reflectivity under Sparsity Constraints}

In the second stage of the algorithm, we aim at estimating the reflectivity vectors based on the obtained wavelet estimate at each sensor. Let us now assume that an estimate of the wavelet is obtained as described in Section 3.1. We will denote this estimate by $\boldsymbol{w}_{j}$. Based on the wavelet estimate $\boldsymbol{w}_{j}$ at each sensor $j$ we can then formulate an optimization problem to recover the reflectivity $\boldsymbol{h}_{j}$. To this end, we refer to system model (4) since here the reflectivity vector $\boldsymbol{h}_{j}$ explicitly occurs in the formulation and the convolution matrix of the wavelet can be constructed based on the node's wavelet estimate $\boldsymbol{w}_{j}$. Based on $\boldsymbol{w}_{j}$ each sensor $j$ constructs an individual convolution matrix $\widehat{\boldsymbol{W}}_{j}$ as an estimate of the original convolution matrix $\boldsymbol{W}$ from (4). The reflectivity vector $\boldsymbol{h}_{j}$ is known to have a sparse structure, i.e., the majority of its entries are zero and only a few elements have a non-zero amplitude [8]. This a priori knowledge can be exploited by including a corresponding sparsity constraint in form of the $\ell_{1}$ norm into the optimization problem. For each sensor $j$, we therefore formulate a least absolute shrinkage and selection operator (LASSO) problem as follows:

$$
\min _{\widetilde{\boldsymbol{h}}_{j}}\left\|\boldsymbol{d}_{j}-\widehat{\boldsymbol{W}}_{j} \widetilde{\boldsymbol{h}}_{j}\right\|_{2}^{2}+\lambda_{\ell 1}\left\|\widetilde{\boldsymbol{h}}_{j}\right\|_{1}, \quad \forall j \in \mathcal{J}
$$

with $\left\|\widetilde{\boldsymbol{h}}_{j}\right\|_{1}=\sum_{l=0}^{L_{\mathrm{R}}-1}\left|\widetilde{h}_{j, l}\right|$ and $\lambda_{\ell 1}$ is a regularization parameter that can be tuned to enhance sparsity of the solution with the price of compromising an optimal fit to the seismic data. To solve problem (12) locally at each sensor $j$ we again employ the ADMM. Thus, for a formulation in the framework of the ADMM we introduce an additional variable $\boldsymbol{r}_{j}$ for the $\ell_{1}$-regularization term and add a constraint that guarantees equivalence to the original problem (12):

$$
\begin{aligned}
& \min _{\left\{\widetilde{\boldsymbol{h}}_{j}, \boldsymbol{r}_{j}\right\}}\left\|\boldsymbol{d}_{j}-\widehat{\boldsymbol{W}}_{j} \widetilde{\boldsymbol{h}}_{j}\right\|_{2}^{2}+\lambda_{\ell 1}\left\|\boldsymbol{r}_{j}\right\|_{1} \\
& \text { s.t. } \widetilde{\boldsymbol{h}}_{j}=\boldsymbol{r}_{j}, \quad \forall j \in \mathcal{J}
\end{aligned}
$$

We solve (13) using [6, Section 6.3] and obtain the following update equations for each sensor $j$ :

$$
\begin{aligned}
\widetilde{\boldsymbol{h}}_{j}^{[k+1]} & =\left(\widehat{\boldsymbol{W}}_{j}^{\top} \widehat{\boldsymbol{W}}_{j}+\rho_{\mathrm{R}} \boldsymbol{I}\right)^{-1}\left(\widehat{\boldsymbol{W}}_{j}^{\top} \boldsymbol{d}_{j}+\rho_{\mathrm{R}}\left(\boldsymbol{r}_{j}^{[k]}-\boldsymbol{v}_{j}^{[k]}\right)\right) \\
\boldsymbol{r}_{j}^{[k+1]} & =S_{\lambda_{\ell 1} / \rho_{\mathrm{R}}}\left(\widetilde{\boldsymbol{h}}_{j}^{[k+1]}+\boldsymbol{v}_{j}^{[k]}\right) \\
\boldsymbol{v}_{j}^{[k+1]} & =\boldsymbol{v}_{j}^{[k]}+\widetilde{\boldsymbol{h}}_{j}^{[k+1]}-\boldsymbol{r}_{j}^{[k+1]}
\end{aligned}
$$

where the variable $\rho_{\mathrm{R}}>0$ is the penalty parameter of the ADMM. For ease of notation we use the same iteration index $k$ here as in the stage of wavelet estimation although it should be noted that these indices are not equivalent to each other. The function $S_{\lambda_{\ell 2} / \rho_{\mathrm{R}}}(\cdot)$ is a soft-thresholding operator defined as $S_{\alpha}(x)=\operatorname{sign}(x) \max (|x|-$ $\alpha, 0)$ and it is applied element-wise to a vector argument. In each iteration $k$ the sparse estimate of the reflectivity vector $\boldsymbol{h}_{j}$ is given by $\boldsymbol{r}_{j}^{[k]}$ after applying the soft-thresholding operator on $\widetilde{\boldsymbol{h}}_{j}^{[k]}+\boldsymbol{v}_{j}^{[k-1]}$. By using (14) each sensor $j$ is able to locally obtain a sparse estimate $\boldsymbol{r}_{j}^{[k]}$ of its reflectivity vector $\boldsymbol{h}_{j}$. Another widely used algorithm to solve LASSO problems is the fast iterative shrinkage-thresholding algorithm (FISTA) [9]. However, in our experiments we noted that 


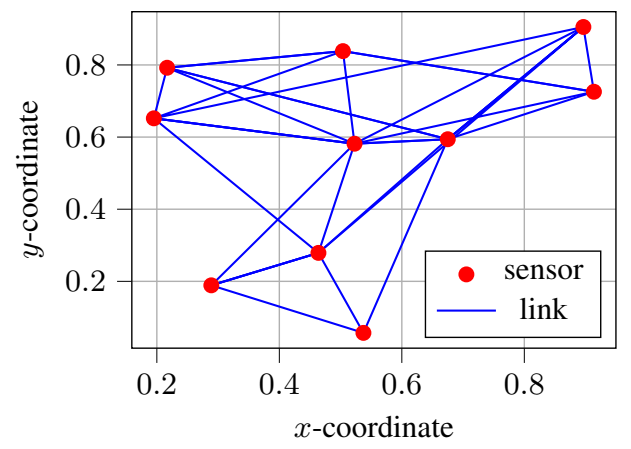

Fig. 1: Considered network topology with $J=10$ sensors.

FISTA requires more iterations than the ADMM to achieve the same estimation performance. For this reason, we selected the ADMM for the estimation of the reflectivities, but note that other algorithms can be also used for this purpose. Algorithm 1 summarizes the whole procedure of the proposed distributed sparse blind deconvolution (D-SBD). Here, we denote the outer iterations by $(k)$ and the inner iterations by $[m],[n]$, respectively. Note that in a further stage the obtained reflectivity estimates need to be processed distributedly among the sensors in order to obtain a subsurface image at each sensor. This stage is left for future work.

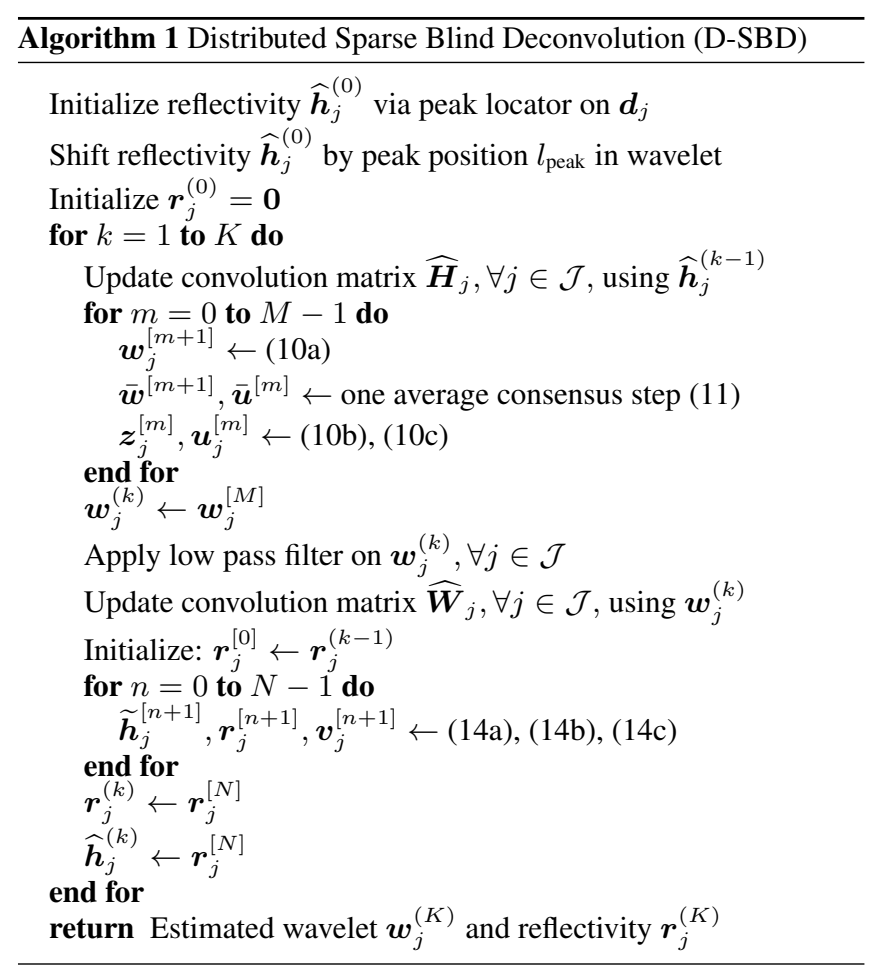

\section{NUMERICAL RESULTS}

To evaluate the performance of our proposed scheme we consider the estimation of synthetically generated reflectivities by a sensor network. To this end, we assume a network of $J=10$ fixed sensors and generate 10 reflectivities, one for each sensor, with a sampling frequency of $500 \mathrm{~Hz}$. The generated reflectivities can be seen in Figure $3 \mathrm{~b}$. The network graph considered in these evaluations is shown in Figure 1. To generate seismic traces at each sensor, we employ a Ricker wavelet with a center frequency of $40 \mathrm{~Hz}$ and a phase shift of $30^{\circ}$. The duration of the wavelet is $T_{\mathrm{W}}=0.1 \mathrm{~s}$ and it is shifted by 16 samples into positive time. The wavelet is convolved with each of the reflectivities and white Gaussian noise of variance $\sigma_{j}^{2}$ is added onto the resulting signal for sensor $j$. We define the signal-tonoise ratio $(\mathrm{SNR})$ via SNR $=10 \log _{10}\left\{\left\|\boldsymbol{s}_{j}\right\|_{2}^{2} /\left(L_{\boldsymbol{s}} \sigma_{j}^{2}\right)\right\}$, where $\boldsymbol{s}_{j} \in \mathbb{R}^{L_{\boldsymbol{s}}}$ is the clean seismic trace for sensor $j$ without noise and $\sigma_{j}^{2}$ is the corresponding noise power. For each trace we assume the same SNR.

As reference schemes we use the SMBD-SPG proposed in [4] and the centralized version of the D-SBD that uses a centralized LS wavelet estimation following (7). We denote the centralized case of the D-SBD as centralized sparse blind deconvolution (C-SBD), here. For all algorithms we use $K=5$ outer iterations. Moreover, the D-SBD uses $M=10$ inner iterations for the distributed wavelet estimation and $N=10$ inner iterations for the reflectivity estimation based on the ADMM LASSO. For the ADMM the penalty parameters are set to $\rho_{\mathrm{W}}=15, \rho_{\mathrm{R}}=1$, the $\mathrm{LS}$ regularization parameter is set to $\lambda_{\ell 2}=0.1$ and the LASSO parameter to $\lambda_{\ell 2}=0.6$. For a fair comparison C-SBD uses the same parameter setup as the D-SBD.

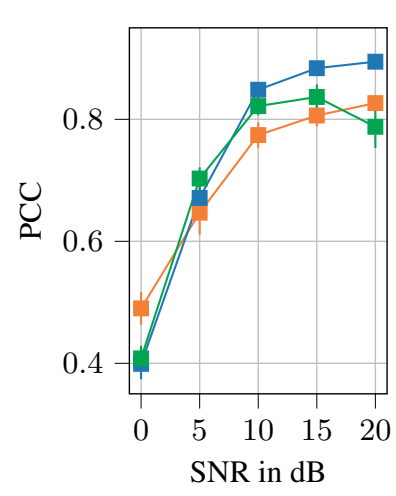

(a) Reflectivity PCC

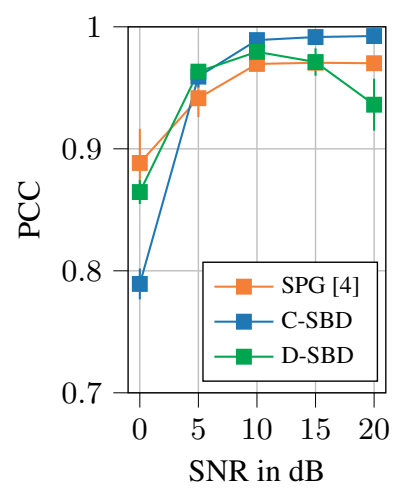

(b) Wavelet PCC
Fig. 2: PCC performance for (a) reflectivity and (b) wavelet estimation.

\subsection{PCC Performance}

As first performance metric we employ the Pearson correlation coefficient (PCC) that gives a similarity measure between two vectors. For vectors $\boldsymbol{x}, \widehat{\boldsymbol{x}}$ of the same dimension it is defined as PCC $=$ $\boldsymbol{x}^{\top} \widehat{\boldsymbol{x}} /\left(\|\boldsymbol{x}\|_{2}\|\widehat{\boldsymbol{x}}\|_{2}\right)$. A PCC value close to one indicates a high similarity between the two vectors. We use the PCC to measure how well our reflectivity and wavelet estimates match their original quantities. Figure 2a depicts the PCC over the SNR for the considered algorithms with respect to the reflectivity estimation where the PCC values are averaged over all sensors and over 20 independent trials. We observe that D-SBD performs close to its central benchmark, the C$\mathrm{SBD}$, and outperforms the SMBD-SPG for $5 \mathrm{~dB} \leq \mathrm{SNR} \leq 15 \mathrm{~dB}$. At $\mathrm{SNR}=20 \mathrm{~dB}$, D-SBD loses in performance and is outperformed by SMBD-SPG. In Figure 2b we compare the PCCs for the wavelet estimate where again the PCC values are averaged over all sensors. For SNR $>5 \mathrm{~dB}$ the C-SBD performs best among the schemes. D-SBD outperforms SMBD-SPG in the mid-SNR range but loses in 


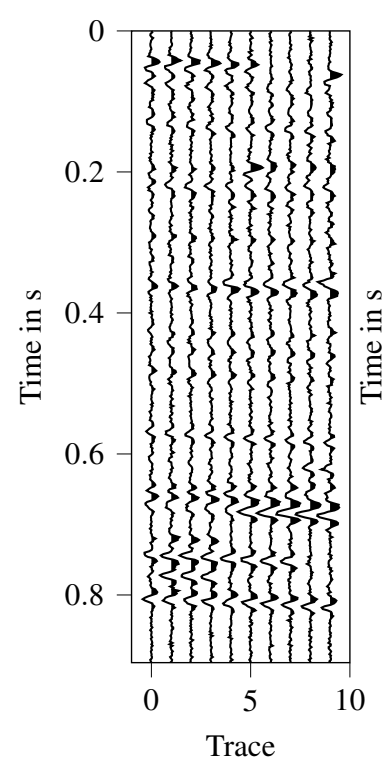

(a) Seismic traces.

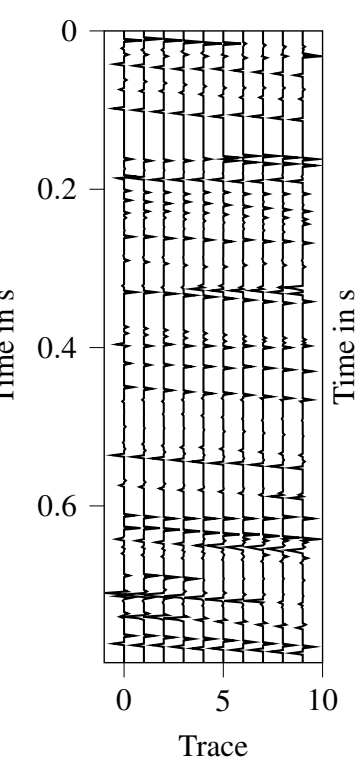

(b) True reflectivities.

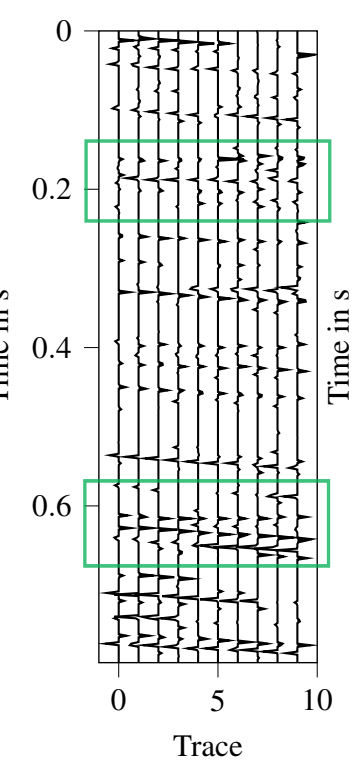

(c) D-SBD

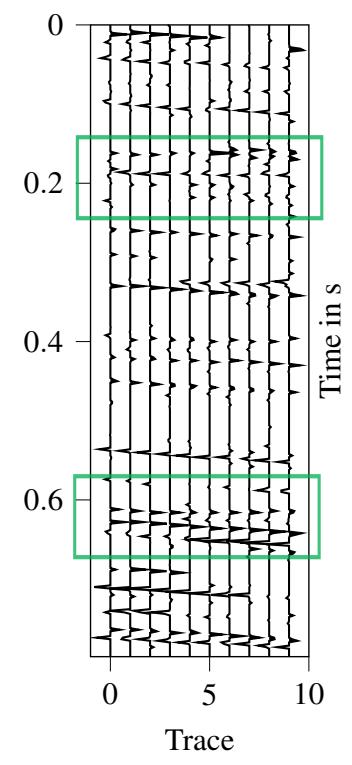

(d) C-SBD.

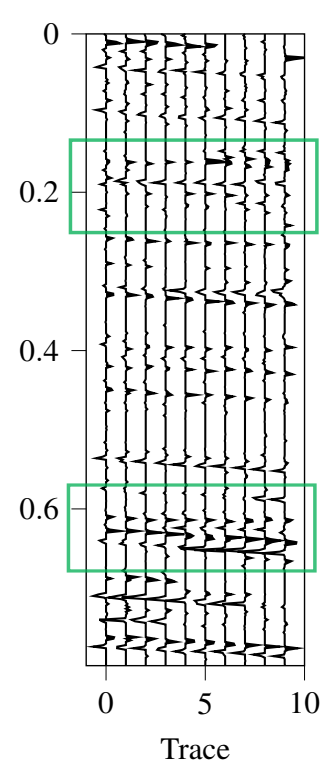

(e) SMBD-SPG [4].

Fig. 3: Example of seismic traces with deconvolved results at $\mathrm{SNR}=10 \mathrm{~dB}$.

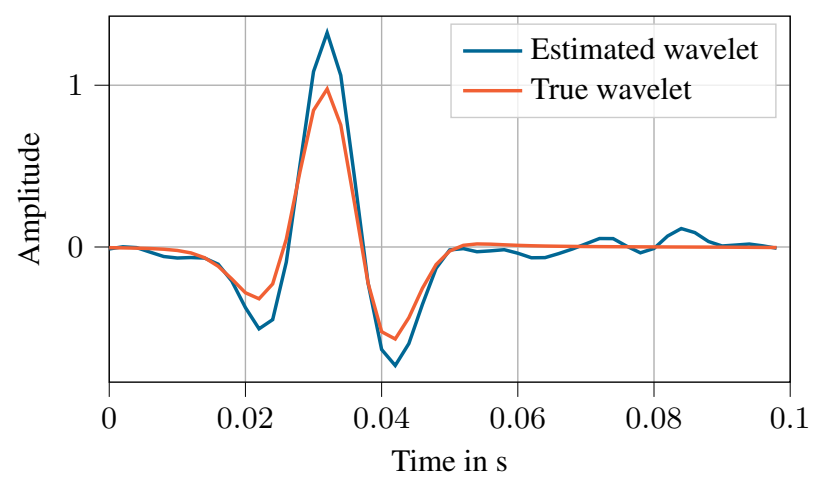

Fig. 4: Estimated wavelet of D-SBD at SNR $=10 \mathrm{~dB}$ after 5 iterations.

performance at high SNR. For a low SNR of $0 \mathrm{~dB}$ the SMBD-SPG obtains the best wavelet estimate. Figure 4 illustrates an exemplary wavelet estimate at one sensor after 5 iterations of the D-SBD at $\mathrm{SNR}=10 \mathrm{~dB}$. One can observe that the peak of the wavelet as well as the sidelobes are recovered. An example of the estimated reflectivities next to the true reflectivity at $\mathrm{SNR}=10 \mathrm{~dB}$ can be seen in Figure 3. Particularly in the marked areas of the plots one can see that D-SBD and C-SBD obtain a spikier reflectivity estimate than SMBD-SPG.

\subsection{EMD Performance}

Using the PCC as a measure to compare sparse vectors as the reflectivities might not be the most suitable metric since it employs an inner product of the vectors. Hence, if two spikes in the vectors appear only a margin apart from each other the corresponding PCC results in a value of zero. Therefore, the PCC does not take into account the distance between two spikes in the vectors. To have a met-

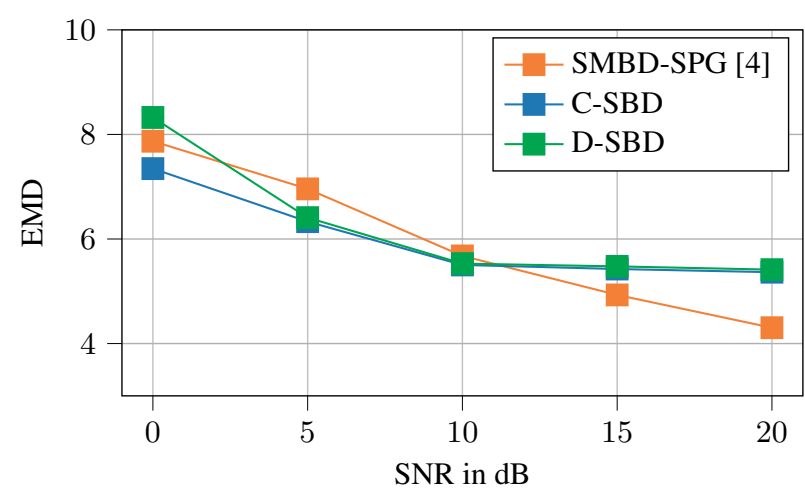

Fig. 5: EMD of estimated reflectivities over SNR.

ric that is aware of the distance between two spikes we employ the Earth Mover's Distance (EMD). The EMD has been proposed as another possible metric for the recovery performance of sparse vectors [10]. Basically, the EMD provides a measure of how much "work" is at least required to transfer one probability distribution into another one. Here, the EMD can be used to quantify the amount of work needed to transfer one sparse vector into another one and therefore it can be used as a similarity measure for sparse vectors. For our evaluations we use the PyEMD package available for Python [11]. More specifically, we use the function emd () which takes three arguments: two sparse vectors under consideration and a distance matrix that is needed to quantify the amount of work needed to move one spike from one entry to another one. The two vectors are the true reflectivity $\boldsymbol{h}_{j}$ and our estimated reflectivity $\boldsymbol{r}_{j}$ at each sensor $j$. For the distance matrix we use the absolute time distance between two elements of the vectors under consideration. Furthermore, we normalize each reflectivity vector to the $\ell_{2}$-norm of the corresponding true reflectivity vector $\boldsymbol{h}_{j}$. 
Figure 5 shows the EMD over the SNR averaged over 20 trials for each SNR value. A lower EMD indicates a higher similarity between true and estimated reflectivities. D-SBD performs very similar to its benchmark C-SBD at $\mathrm{SNR}=0 \mathrm{~dB}$. In the low SNR region C-SBD and D-SBD tend to outperform SMBD-SPG. However, for higher SNRs both C-SBD and D-SBD performance flattens out while SMBD-SPG achieves a lower EMD. This behavior is due to a fixed LASSO parameter $\lambda_{\ell 1}$ used in both D-SBD and C-SBD over the whole SNR range. SMBD-SPG uses a basis pursuit denoising solver to obtain sparse estimates. Here, the corresponding threshold for the solver is set depending on the true noise power $\sigma_{j}^{2}$. Hence, SMBD-SPG adapts its sparse recovery to the current SNR and therefore achieves lower EMD values for higher SNRs.

\subsection{Performance over LASSO Parameter}

As a last result we examine the estimation performance in dependence of the LASSO parameter $\lambda_{\ell 1}$ for the D-SBD. Figure 6a and $6 \mathrm{~b}$ show the PCC and the EMD over different values of $\lambda_{\ell 1}$, respectively. The performance is averaged over 20 independent trials. At low SNR $\lambda_{\ell 1}=1$ leads to the highest PCC whereas for higher SNRs a lower value of $\lambda_{\ell 1}=6 \mathrm{~dB}$ is appropriate. Surprisingly, the PCC at SNR $=20 \mathrm{~dB}$ does not outperform the performance at $\mathrm{SNR}=10 \mathrm{~dB}$ over the whole range of $\lambda_{\ell 1}$. Hence, it seems that the choice of the ADMM penalty parameter $\rho_{\mathrm{W}}$ for the wavelet estimation requires an additional adaptation to the SNR. In general, with an increasing SNR the parameter $\lambda_{\ell 1}$ should be reduced. This can be also observed for the EMD in Figure 6b. Here, the curves again suggest that for high SNRs low values of $\lambda_{\ell 1}$ are appropriate and vice versa. However, the values leading to the corresponding minimal EMD are quite different compared to the PCC result. For instance, at SNR $=20 \mathrm{~dB}$ a value of $\lambda_{\ell 1}=0.1$ leads to the smallest EMD which does not coincide with the results for the PCC. Since PCC and EMD differ in their computation the corresponding values for $\lambda_{\ell 1}$ do not coincide either. Both results with regard to PCC and EMD show that the performance of the reflectivity estimation highly depends on the LASSO parameter $\lambda_{\ell 1}$. Therefore, setting $\lambda_{\ell 1}$ appropriately with respect to the SNR is important for a satisfactory reflectivity recovery.

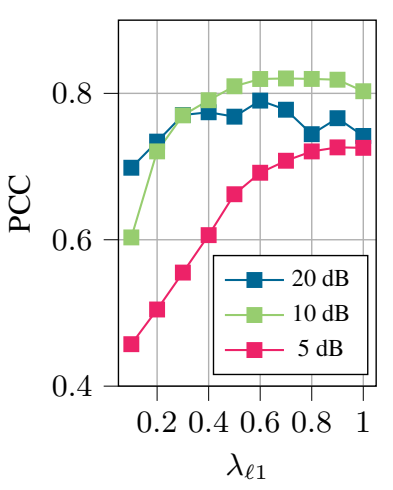

(a) PCC over $\lambda_{\ell 1}$.

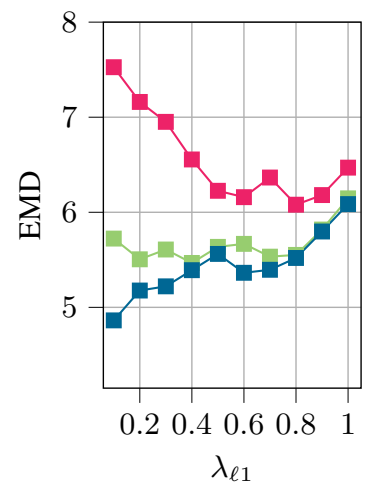

(b) EMD over $\lambda_{\ell 1}$.
Fig. 6: Performance of D-SBD over $\lambda_{\ell 1}$ for (a) PCC and (b) EMD.

\section{CONCLUSION AND FUTURE WORK}

We proposed a distributed algorithm for the blind deconvolution of seismic signals under sparsity constraints. To this end, we employed a distributed wavelet estimation and a local reflectivity estimation. The wavelet estimation is realized by a LS consensus-based ADMM approach. The reflectivity estimation is done locally at each sensor using an ADMM-based LASSO scheme. Performance evaluations show that our proposed scheme performs close to its central benchmark scheme and outperforms state-of-the-art sparse blind deconvolution in terms of the PCC in mid SNR regions. We also investigated the EMD performance and found that for higher SNRs the performance of our proposed scheme flattens out. This behavior is due to a fixed LASSO and ADMM penalty parameter. For future work, an adaptive parameter mechanism shall be realized in the framework of Bayesian learning to improve the performance and robustness of the algorithm. Furthermore, the proposed scheme will be investigated for the deconvolution of real seismic data.

\section{REFERENCES}

[1] Hua-Wei Zhou, Practical Seismic Data Analysis, Cambridge University Press, 2014.

[2] WeiJia Sun, Liang Zhao, Yong Wei, and Li-Yun Fu, "Detection of seismic events on Mars: a lunar perspective," Earth and Planetary Physics, vol. 3, no. 4, pp. 1-8, 2019.

[3] Kenji Nose-Filho, Andre Kazuo Takahata, Renato Lopes, and Joao Marcos Travassos Romano, "Improving sparse multichannel blind deconvolution with correlated seismic data: Foundations and further results," IEEE Sig. Proc. Mag., vol. 35, no. 2, pp. 41-50, 2018.

[4] Naveed Iqbal, Entao Liu, James H. McClellan, and Abdullatif A. Al-Shuhail, "Sparse multichannel blind deconvolution of seismic data via spectral projected-gradient," IEEE Access, vol. 7, pp. 23740-23751, 2019.

[5] Ali Sayed, "Adaptation, Learning, and Optimization over Networks," Foundations and Trends ${ }^{\circledR}$ in Machine Learning, vol. 7, no. 4-5, pp. 311-801, 2014.

[6] Stephen Boyd, Neal Parikh, Eric Chu, Borja Peleato, and Jonathan Eckstein, "Distributed optimization and statistical learning via the alternating direction method of multipliers," Foundations and Trends in Machine Learning, vol. 3, no. 1, pp. 1-122, 2010.

[7] L Xiao, S Boyd, and S. Lall, "A scheme for robust distributed sensor fusion based on average consensus," in Proceedings of the 4th international symposium on Information processing in sensor networks. 2005, vol. pages, p. 9, IEEE Press.

[8] Ralph A. Wiggins, "Minimum entropy deconvolution," Geoexploration, vol. 16, pp. 21-35, 1978.

[9] Amir Beck and Marc Teboulle, "A fast iterative shrinkagethresholding algorithm," Society for Industrial and Applied Mathematics Journal on Imaging Sciences, vol. 2, no. 1, pp. 183-202, 2009.

[10] A. Lavrenko, F. Römer, G. Del Galdo, and R. Thoma, "On the earth mover's distance as a performance metric for sparse support recovery," in IEEE GlobalSIP, 2016, pp. 1368-1372.

[11] Ofir Pele and Michael Werman, "Fast and robust earth mover's distances," in IEEE International Conference on Computer Vision, September 2009, pp. 460-467. 